\title{
Nucleocytoplasmic transport of nucleocapsid proteins of enveloped RNA viruses
}

\author{
Wahyu N. Wulan 1,2, Deborah Heydet', Erin J. Walker', Michelle E. Gahan ${ }^{2}$ and \\ Reena Ghildyal ${ }^{1,2 *}$ \\ ${ }^{1}$ Centre for Research in Therapeutic Solutions, University of Canberra, Bruce, ACT, Australia, ${ }^{2}$ Faculty of Education, \\ Science, Technology and Mathematics, University of Canberra, Bruce, ACT, Australia
}

OPEN ACCESS

Edited by:

Akio Adachi,

Tokushima University Graduate

School, Japan

Reviewed by:

Takashi Irie,

Hiroshima University, Japan

Masato Tsurudome,

Mie University Graduate School of Medicine, Japan

Fumitaka Momose

Kitasato University, Japan

*Correspondence:

Reena Ghildyal,

Centre for Research in Therapeutic Solutions and Faculty of Education,

Science, Technology

and Mathematics, University of Canberra, Room 3D51, Bruce, ACT 2617, Australia

reena.ghildyal@canberra.edu.au

Specialty section:

This article was submitted to

Virology,

a section of the journa

Frontiers in Microbiology

Received: 27 February 2015

Accepted: 19 May 2015

Published: 02 June 2015

Citation:

Wulan WN, Heydet D, Walker EJ,

Gahan ME and Ghildyal R (2015)

Nucleocytoplasmic transport of nucleocapsid proteins of enveloped

RNA viruses.

Front. Microbiol. 6:553. doi: 10.3389/fmicb.2015.00553
Most viruses with non-segmented single stranded RNA genomes complete their life cycle in the cytoplasm of infected cells. However, despite undergoing replication in the cytoplasm, the structural proteins of some of these RNA viruses localize to the nucleus at specific times in the virus life cycle, primarily early in infection. Limited evidence suggests that this enhances successful viral replication by interfering with or inhibiting the host antiviral response. Nucleocapsid proteins of RNA viruses have a well-established, essential cytoplasmic role in virus replication and assembly. Intriguingly, nucleocapsid proteins of some RNA viruses also localize to the nucleus/nucleolus of infected cells. Their nuclear function is less well understood although significant advances have been made in recent years. This review will focus on the nucleocapsid protein of cytoplasmic enveloped RNA viruses, including their localization to the nucleus/nucleolus and function therein. A greater understanding of the nuclear localization of nucleocapsid proteins has the potential to enhance therapeutic strategies as it can be a target for the development of live-attenuated vaccines or antiviral drugs.

Keywords: enveloped RNA viruses, nucleocapsid protein, nuclear localization, nucleolar localization, antiviral responses

\section{Introduction}

The nucleocapsid protein of RNA viruses is essential for virus assembly, encapsidating the genomic RNA in preparation for packaging in the virion (Hunter, 2007). In positive strand RNA viruses (PSVs), the capsid or nucleocapsid protein is the major, often the only structural protein. In negative strand RNA viruses (NSVs), the nucleocapsid protein has an essential role in viral replication and transcription (Whelan, 2013) in addition to its role in assembly. For most enveloped RNA viruses, both replication and assembly occur in the cytoplasm (with the exception of orthomyxovirus, bornavirus, retroviruses, and hepatitis delta virus whose replication occurs in the nucleus; Whelan, 2013). Interestingly, the nucleocapsid protein (variously named N, NP, NC, or C protein) of many cytoplasmic RNA viruses (PSVs and NSVs) transiently localizes to the nucleus and/or nucleolus. There is limited information about the nuclear function of cytoplasmic RNA virus nucleocapsid proteins, but evidence suggests supporting roles in successful viral replication by interfering with or inhibiting the host antiviral response (Ahmed and Lyles, 1998; Ahmed et al., 2003; Lee et al., 2006; Takayama et al., 2012) as has been described for several RNA virus structural proteins. 
The nucleocapsid protein of mouse hepatitis virus (MHV, Coronaviridae) and infectious bronchitis virus (IBV, Coronaviridae) localizes to the nucleolus of infected cells where it may regulate/delay cell cycle progression (Wurm et al., 2001), while nuclear localization of vesicular stomatitis virus (VSV, Rhabdoviridae) M, rabies (Rhabdoviridae) $\mathrm{P}$, and Measles virus (MV, Paramyxoviridae) N protein (Ahmed et al., 2003; Schnell et al., 2010; Takayama et al., 2012) is associated with the pathogenesis of viral infection. Nuclear localization of the nucleocapsid protein of porcine respiratory and reproductive syndrome virus (PRRSV, Arteriviridae) appears to be essential for optimal virus replication and inhibition of cellular antiviral processes (Lee et al., 2006); in an analogous fashion, the successful production of infectious progeny of respiratory syncytial virus (RSV, Paramyxoviridae) and Nipah virus (Paramyxoviridae) requires nuclear localization of the matrix (M) protein (Ghildyal et al., 2005, 2009; Wang et al., 2010).

The aim of this review is to summarize current literature on the role of nuclear/nucleolar localization of nucleocapsid proteins of cytoplasmic PSVs and NSVs, which may lead to similar investigations in other related virus families. Most of our knowledge in this area derives from studies of viruses belonging to the Coronaviridae, Arteriviridae, Flaviviridae, and Paramyxoviridae families and these will be the main focus (Table 1), with reference to other virus families when required. Coronaviridae and Arteriviridae are classified together into the order Nidovirales; for the sake of simplicity and due to the close relationship among these viruses, both families will be discussed together.

The location of the nucleocapsid gene within the genome of selected viruses from the named families is shown in Figure 1. The nucleocapsid [N, NC, or C (core or capsid)] gene is in the first position ( $3^{\prime}$ end) in Paramyxovirus genomes and $\left(5^{\prime}\right.$ end) in Flavivirus and Hepacivirus genomes. The nucleocapsid gene of Coronavirus and Arterivirus is in the last position ( $3^{\prime}$ end) of these genomes, but the first gene to be copied into a negative sense subgenomic mRNA template for mRNA transcription.

\section{The Cytoplasmic Role of RNA Virus Nucleocapsid Protein}

The nucleocapsid protein of RNA viruses is a structural protein found in a complex with the genomic RNA. The role of genome encapsidation varies across PSVs and NSVs, but usually comprises one or more of the following functions; [1] to protect the genome from cellular nucleases, [2] to form the ribonucleoprotein transcriptase complex, and [3] to package the genome into new infectious particles prior to budding.

\section{Nucleocapsid Protein of NSVs}

The NSV nucleocapsid protein protects genomic viral RNA against ribonuclease degradation and high salt concentration, as well as maintaining the rigidity of the RNA structure following cycles of folding/dissociation/refolding during the replication cycle (Lynch and Kolakofsky, 1978; Mir and Panganiban, 2006; Raymond et al., 2010). The nucleocapsid protein is the driving force for the formation of ribonucleoprotein (RNP) complexes that are the replicative/transcriptive unit, as well as for assembly and packaging into the virion. These functions are facilitated through its propensity to self-associate and ability to interact with other RNP components, including RNA and viral polymerase subunits (Becker et al., 1998; DiCarlo et al., 2007; Green et al., 2011; Sun et al., 2012). Nucleocapsid protein has an inherent ability to interact with the phosphoprotein (P; polymerase subunit and cofactor). This interaction is particularly important because association between nucleocapsid and RNA is not specific; instead, specificity is conferred via the nucleocapsid-phosphoprotein interaction, such that RNA associated with the nucleocapsid-phosphoprotein complex is the form recognized by RNA polymerase (Myers et al., 1999; Longhi, 2009). Additionally, the NSV phosphoprotein acts as a chaperone for the nucleocapsid protein restricting nonspecific encapsidation of non-viral RNA (Curran et al., 1995; Errington and Emmerson, 1997). The nucleocapsid protein of VSV, in association with the $\mathrm{P}$ protein, is required for genome encapsidation and transcription (Zhang et al., 2008; Morin and Whelan, 2014) with mutations in its C-terminus resulting in reduction of viral particle production in cell culture (Heinrich et al., 2012). In RSV and Sendai virus (Paramyxoviridae), it is the $\mathrm{N}$-terminal of the nucleocapsid protein that plays a similar role in genome encapsidation (Buchholz et al., 1993; Myers and Moyer, 1997; Khattar et al., 2000).

\section{The Nucleocapsid/Capsid/Core Protein of PSVs}

Similar to the NSV nucleocapsid protein, the nucleocapsid/ capsid/core protein of PSVs binds tightly to the genomic RNA, drives RNA packaging and sometimes, serves as a nucleic acid chaperone. Flavivirus (Flaviviridae) $\mathrm{C}$ protein plays an important role during assembly and budding of infectious particles. Mutations in $\mathrm{C}$ protein lead to the inhibition of infectious particle release while causing increased production of defective particles (Jones et al., 2011). The hepatitis C virus (HCV, Flaviviridae) core protein and the $\mathrm{N}$ protein of coronaviruses severe acute respiratory syndrome coronavirus (SARS-CoV) and transmissible gastroenteritis virus (TGEV) are potent RNA chaperones that can resolve RNA misfolding and promote annealing of complementary sequences (Rajkowitsch et al., 2007; Zuniga et al., 2007; Sharma et al., 2010). Most of the evidence for chaperone activity is derived from in vitro investigations and although these proteins clearly have the ability to function as RNA chaperones, the importance of this function in infection is not clear.

\section{Non-Encapsidation Roles of Nucleocapsid Proteins}

The N/NC/C proteins of both NSVs and PSVs have functions in addition to their RNA stabilizing, encapsidating and, in 
TABLE 1 | Classification of enveloped RNA viruses discussed in the review.

\begin{tabular}{|c|c|c|c|}
\hline Family & Sub-family & Genus & Species \\
\hline \multirow[t]{2}{*}{ Flaviviridae } & & Flavivirus & Dengue virus, Japanese Encephalitis virus (JEV), West Nile virus \\
\hline & & Hepacivirus & Hepatitis C virus \\
\hline \multirow[t]{3}{*}{ Coronaviridae } & Coronavirinae & Alphacoronavirus & Transmissible gastroenteritis virus (TGEV) \\
\hline & & Betacoronavirus & $\begin{array}{l}\text { Severe acute respiratory syndrome coronavirus (SARS-CoV), mouse hepatitis } \\
\text { virus }\end{array}$ \\
\hline & & Gammacoronavirus & Infectious bronchitis virus \\
\hline Arteriviridae & & Arterivirus & $\begin{array}{l}\text { Lactose dehydrogenase elevating virus, Equine arterivirus, Porcine respiratory } \\
\text { and reproductive syndrome virus }\end{array}$ \\
\hline \multirow[t]{4}{*}{ Paramyxoviridae } & Paramyxovirinae & Morbillivirus & Measles virus, Canine distemper virus (CDV), Rinderpest virus \\
\hline & & Henipavirus & Nipah virus \\
\hline & & Respirovirus & Sendai virus \\
\hline & Pneumovirinae & Pneumovirus & Respiratory syncytial virus \\
\hline
\end{tabular}

the case of NSVs, viral transcriptase activities. The flavivirus core protein interacts with several host cell proteins and is implicated in disease development through its roles in apoptotic pathways as well as regulation of the innate immune response (Urbanowski et al., 2008). Similarly, the coronavirus N protein interferes with cellular antiviral responses (McBride et al., 2014). The $\mathrm{N}$ protein of morbilliviruses (Paramyxoviridae) has been shown to regulate interferon signaling via interference with the signal transducer and activator of transcription (STAT) signaling pathway (Takayama et al., 2012). Increasing literature suggests that at least some of the non-encapsidating functions of the nucleocapsid proteins of NSVs and PSVs derive from their ability to localize to the nucleus or nucleolus of the infected cells.

\begin{tabular}{l} 
Coronavirus: (+)ssRNA, $27-32 \mathrm{~kb}$ \\
\hline Pol-1a
\end{tabular}

\section{Nuclear Localization of Nucleocapsid Proteins of RNA Viruses}

Despite undergoing replication in the cytoplasm, the nucleocapsid protein of several NSVs and PSVs localizes in the nucleus or nucleolus of infected cells during infection. This nuclear localization usually takes place early in the infectious cycle as soon as the nucleocapsid protein is translated, possibly to perform non-structural functions, followed by return to the cytoplasm in the late stage of infection to participate in assembly (Tijms et al., 2002; Yoo et al., 2003; Sato et al., 2006). Although some progress has been made in our understanding of the nucleocytoplasmic shuttling of several nucleocapsid proteins, thus far, no definitive single molecule studies have been undertaken; such studies will be important to elucidate the finer details of nuclear transport pathways in the future.

\section{Nucleocytoplasmic Transport}

Eukaryotic cells sequester their genome in the nucleus, which is surrounded by the double lipid bilayer structure of the nuclear envelope (NE). The only avenue for transport into and out of the nucleus is via the NE-embedded nuclear pore complexes (NPCs) that are made up of over 40 different proteins called nucleoporins (Nups). Although diffusion of molecules $<55 \mathrm{kDa}$ can occur, most transport through the NPC is mediated by members of the importin superfamily, which recognize nuclear localization sequences (NLSs) or nuclear export sequences (NESs) on cargo molecules for transport into and out of the nucleus, respectively (Ghildyal et al., 2005, 2009; Cardarelli et al., 2008; Cohen et al., 2011). NLSs are usually short stretch(es) of basic residues (monopartite NLS, e.g., that of the simian virus $40 \mathrm{~T}$ antigen) which may be separated by $10-15$ amino acids (bipartite NLS, e.g., that of nucleophosmin), while the most well characterized NES is the Leucine-rich motif recognized by the exportin CRM-1 (e.g., the NES in HIV Rev protein). Importins function by binding NLSs and docking transiently at various "FG" (Phenylalanine-Glycine repeat containing) Nups within the NPC to effect translocation through it, followed by release within the nucleus facilitated by the guanine nucleotide binding protein 
Ran (Gorlich and Kutay, 1999). The best studied nuclear import pathway is mediated by the importin- $\alpha / \beta 1$ heterodimer, where importin- $\alpha$ recognizes and directly binds to the NLSs of the cargo, and importin- $\beta 1$ mediates binding of the import complex to Nups (Macara, 2001). However, nuclear import can be effected by direct action of importin- $\beta 1$ or homologs thereof, without a requirement for importin- $\alpha$ (Fulcher and Jans, 2011). In all cases, release within the nucleus occurs through binding of Ran, in its guanosine triphosphate (GTP)-bound form, to importin- $\beta 1$, or homologs, to effect dissociation of the import complex. Nuclear export is analogous to nuclear import, wherein cargo molecules containing NESs bind importin- $\beta$ homologs, such as CRM-1, in complex with Ran in its GTP bound state and are transported out of the nucleus; release in the cytoplasm is facilitated by Ran hydrolysis of GTP to guanosine diphosphate (GDP), which leads to dissociation of the export complex (Macara, 2001).

Localization to the nucleolus is less well defined, but is most likely regulated by cargoes interacting with nucleolar residents, possibly the so-called hub proteins (Emmott and Hiscox, 2009). Nucleolar localization signals (NoLSs), similar to NLSs, are short stretches of basic proteins, however, there is a high level of variability in the NoLSs described to date (de Melo et al., 2013). NoLSs are not capable of directing cargo into the nucleus, although very often they overlap the NLS sequences. Interestingly, CRM-1 has been shown to play an important role in nucleolar localization of small nuclear and nucleolar RNA molecules via complex binding mechanisms that lead to masking/unmasking of the NoLS (Verheggen and Bertrand, 2012).

\section{Coronaviridae and Arteriviridae}

Viruses in the Coronaviridae family possess a positive $(+)$ ssRNA genome and cluster into two subfamilies including Coronavirinae which includes the human coronaviruses, and six genera. The viruses of Arteriviridae family are also positive $(+)$ ssRNA viruses and grouped into one genus, Arterivirus.

The nucleocapsid proteins of viruses belonging to genus Arterivirus localize to the nucleus, as has been shown for lactate dehydrogenase-elevating virus (LDV; Mohammadi et al., 2009), equine arteritis virus (EAV; Tijms et al., 2002) and PRRSV (Rowland et al., 1999). Nuclear import of the LDV nucleocapsid protein is facilitated by a monopartite NLS motif, ${ }^{38} \mathrm{KKKK}^{41}$ (Figure 2A), probably mediated by the importin- $\alpha / \beta$ complex (Mohammadi et al., 2009), and whilst the EAV nuclear import has not been clearly defined, nuclear export is facilitated by CRM-1 (Tijms et al., 2002). PRRSV nucleocapsid protein possesses NLS, nucleolar retention/localization signal (NoRS/-LS), and NES motifs that facilitate independent nucleocytoplasmic transport and accumulation in the nucleoli of infected cells (Rowland et al., 1999; Yoo et al., 2003). The NLS motifs that are located in amino acid residues 10-13 (NLS1) and 41-47 (NLS2) facilitate nuclear import via interaction with importin- $\alpha / \beta$, while the NES motif is located in amino acid residues 106-117 and works with CRM-1 to facilitate nuclear export (Pei et al., 2008). Disruption of nuclear/nucleolar localization of PRRSV nucleocapsid protein has been shown not to affect the capability to produce infectious particles in MARC-145 cells (monkey kidney cells permissive

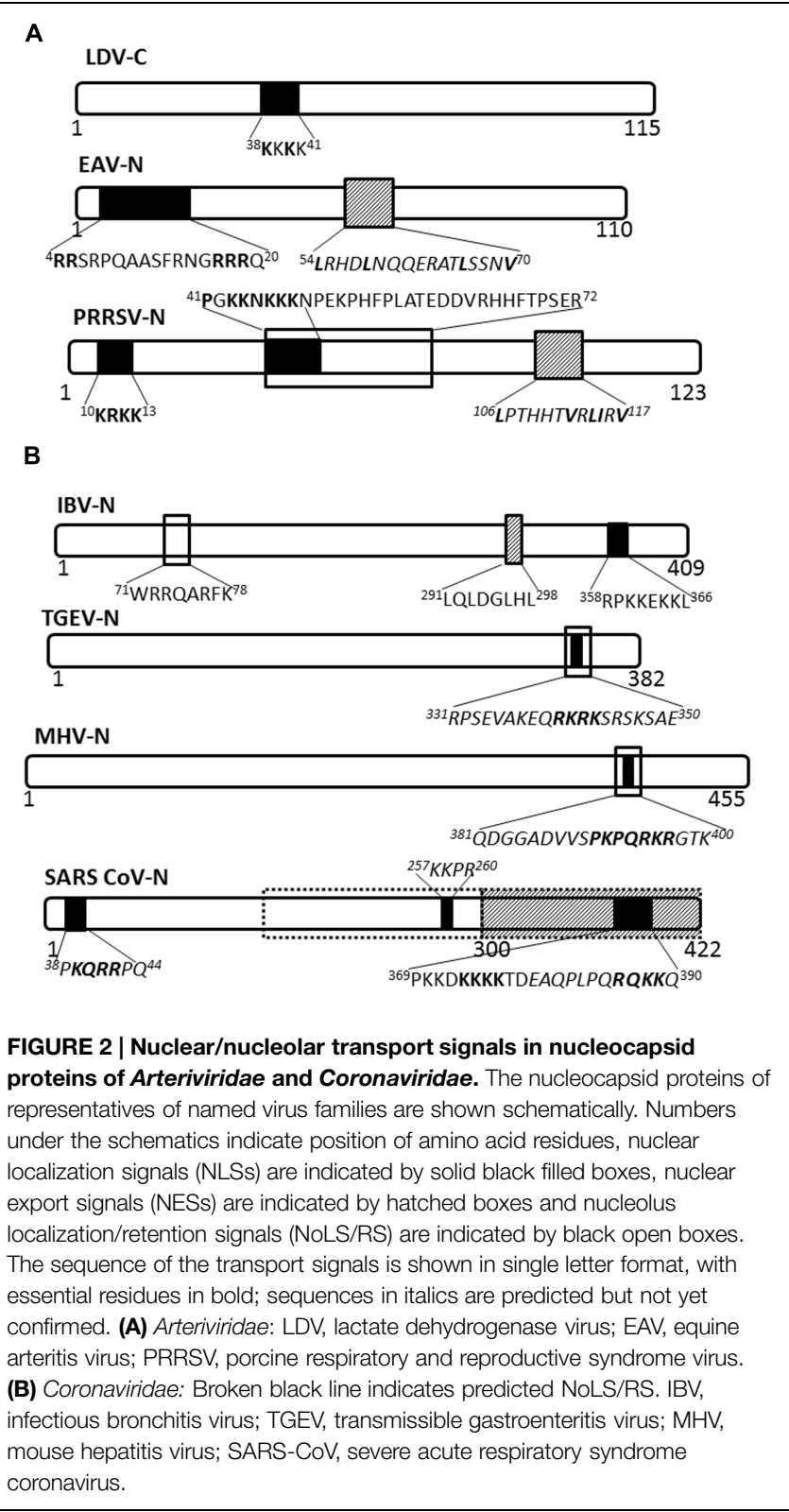

to PRRSV infection), however, it did result in attenuated viral replication and induced a higher titer of neutralizing antibody in pigs. This suggests the nucleocytoplasmic localization of PRRSV nucleocapsid protein has an important role in pathogenesis of PRRSV infection (Lee et al., 2006; Pei et al., 2008).

Nucleocapsid proteins of coronaviruses IBV and $\mathrm{MHV}$ localize to the nucleolus of infected cells facilitated by NLSs, NoRS/-LS, and an NES (Hiscox et al., 2001; Wurm et al., 2001; Chen et al., 2002; Reed et al., 2006; Figure 2B). In the nucleolus the nucleocapsid protein may modulate cell division, as evidenced by the fact that cells expressing coronavirus nucleocapsid protein appear to be arrested in the G2/M phase of the cell cycle (Chen et al., 2002; Ding et al., 2014a; McBride et al., 2014). Delay in the cell cycle may promote conditions suitable for virus replication and assembly. 
The nucleocapsid protein of IBV has been shown to associate with fibrillarin and nucleolin in the nucleolus (Hiscox et al., 2001). In particular, as also observed for $\mathrm{MHV}$ and TGEV nucleocapsid proteins, it causes G2 arrest of the cell cycle that leads to reorganized fibrillarin distribution, where the interaction and/or redistribution are associated with a delay in cell growth (Hiscox et al., 2001; Chen et al., 2002; Ding et al., 2014a).

The nucleolus is a multifunctional nuclear substructure that is central to the normal operations of a cell, with important roles in ribosome synthesis and assembly, cell cycle regulation, transcription regulation, cell senescence and sensing and response to stress (Bartova et al., 2010; Shaw and Brown, 2012; Golomb et al., 2014). The nucleolar proteome consists of more than 500 proteins identified so far; some are long term residents, while others shuttle between the nucleolus, nucleoplasm, cytoplasm, and cell membrane (Andersen et al., 2002; Lam et al., 2005) depending on the metabolic state of the cell. Fibrillarin, nucleolin, and nucleophosmin are major nucleolar proteins that have been studied extensively. Fibrillarin is involved in many post-transcription processes and ribosome assembly; nucleolin serves crucial roles in pre-rRNA processing and may regulate transcription; nucleophosmin is implicated in ribosome assembly, nucleocytoplasmic shuttling and may regulate rDNA transcription [(Hiscox, 2002) and references therein].

The SARS-CoV nucleocapsid protein is predominantly cytoplasmic in infected cells and in cells transfected to express the full length protein. Interestingly, the protein sequence contains three putative NLSs and a putative NES (Figure 2B); the latter is non-functional (You et al., 2005, 2007). A bipartite NLS and putative NoLS appear to be functional but are masked in the context of the full length protein by a dominant CRM1 -independent NES in the C-terminus. Additionally, SARS $\mathrm{CoV}$ nucleocapsid cytoplasmic localization is facilitated by interaction with 14-3-3 (tyrosine 3-monooxygenase/tryptophan 5-monooxygenase activation protein; Macara, 2001; Surjit et al., 2005). Thus, the SARS-CoV nucleocapsid protein, to the best of our knowledge, does not localize to the nucleus or the nucleolus of infected cells, in contrast to the nucleocapsid proteins of other coronaviruses studied.

\section{Paramyxoviridae}

Nucleocapsid proteins of some viruses in the Paramyxoviridae family have been shown to undergo transient nuclear localization. The nucleocapsid proteins of canine distemper virus (CDV) and rinderpest virus (RPV) possess conserved NLS and NES motifs; the NES is CRM-1 independent, despite being a Leucine-rich motif (Sato et al., 2006; Figure 3). MV infected cells contain nuclear inclusion bodies (composed of $\mathrm{N}$, phosphoprotein and large polymerase; Robbins and Eagle, 1985). More importantly, the nuclear translocation of MV nucleocapsid protein also affects the host immune response by inhibiting the nuclear translocation of STATs, causing disruption of the IFN $-\alpha / \beta$ and IFN $-\gamma$ signaling pathways (Takayama et al., 2012). The NLS motif is contained within ${ }^{70}$ TGILISILSLF ${ }^{80}$; while the NES is contained within residues ${ }^{425}$ SENELPRLGGKEDRRV ${ }^{440}$ (Sato et al., 2006).

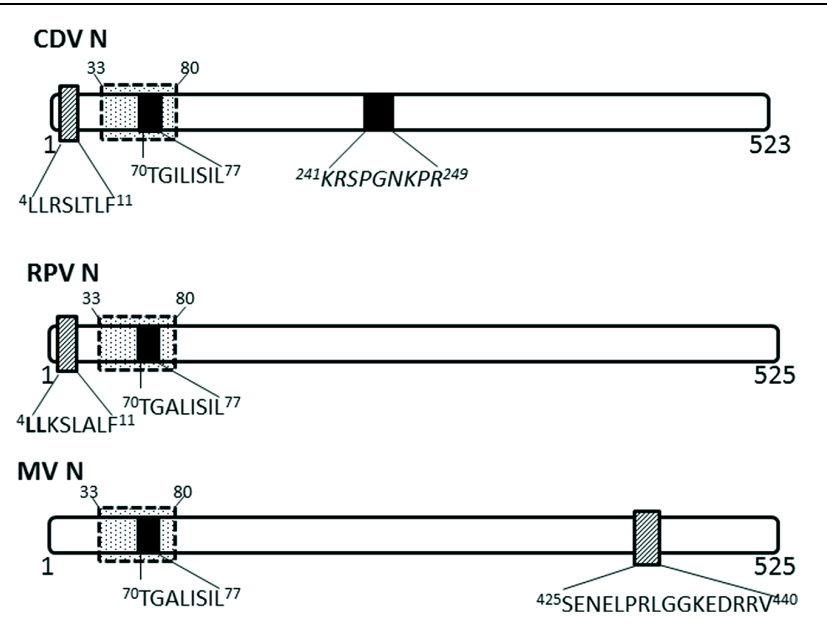

FIGURE 3 | Nuclear transport signals in nucleocapsid proteins of Paramyxoviridae. The NLSs and NESs are indicated as in Figure 2; a dashed-line box with dots indicates flanking sequences that have been shown to have a role in nuclear import and retention. CDV, canine distemper virus; RPV, Rinderpest virus; MV, measles virus.

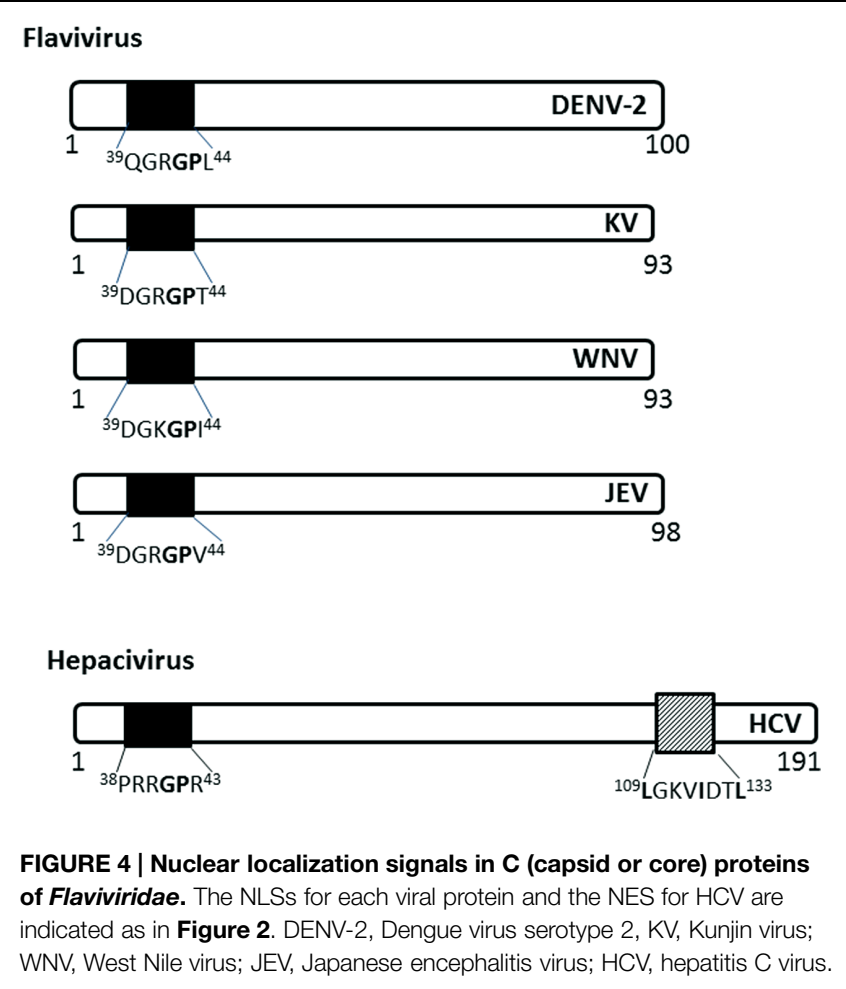

\section{Flaviviridae}

The capsid (C) protein of several members of the Flaviviridae family localize to the nucleus of infected cells using NLSs (Figure 4). The Flavivirus $\mathrm{C}$ protein is small enough to diffuse across the NE; however, studies have shown that it utilizes active nuclear transport pathways to localize to the nucleus.

The $\mathrm{C}$ protein of Dengue virus localizes to the nucleus of infected mammalian cells, starting at $6 \mathrm{~h}$ post-infection (h.p.i) 
and remains at high levels throughout the course of infection (up to 72 h.p.i; Sangiambut et al., 2008). The C protein does not localize to the nucleus of mosquito cells, possibly due to the different availability of carrier proteins or the cellular components involved in nuclear translocation.

The C (core) protein of the Japanese encephalitis virus (JEV) has been shown to localize into the nucleus and associate with the phosphoprotein B23 in the nucleolus (Tsuda et al., 2006). The nuclear localization is required for successful replication in mammalian cell lines and is determined by amino acid residues ${ }^{42} \mathrm{GP}^{43}$ of the NLS, with $\mathrm{G}^{42}$ being the major determinant (Mori et al., 2005). ${ }^{42} \mathrm{GP}^{43}$ substitution into $\mathrm{A}^{42-43}$ eliminates the nuclear localization ability in both mosquito and mammalian cell lines and decreases infectious virus production in mammalian cell lines and in infected mice. The GP motif is conserved in Dengue, Kunjin, and Hepatitis C virus C proteins (Mori et al., 2005).

Hepatitis C virus $C$ protein has been found in the nucleus in the C-terminally truncated form, particularly in hepatocytes isolated from chronic HCV patients (Falcon et al., 2003), suggesting a possible role in persistence of HCV infection, which often leads to hepatocellular carcinoma. The nuclear localization of HCV C is dependent on importin- $\alpha$, which recognizes NLS motifs contained within the residues 5-13 (PKPQRKTKR), 38-43 (PRRGPR), and 58-71 (PRGRRQPIPKARRP; Suzuki et al., 2005). HCV C also has a functional CRM-1 dependent NES in residues 109-133 (Cerutti et al., 2011). The nuclear localization has been observed early in infection ( $\sim$ from 6 h.p.i), similar to that of Dengue C protein, and disruption of the nucleocytoplasmic translocation process reduces the production of infectious particles (Cerutti et al., 2011).

\section{Nuclear Functions of the Nucleocapsid Proteins of RNA Viruses}

Based on current literature and our understanding of nucleocytoplasmic trafficking in the context of virus infection, it can be proposed that the nuclear accumulation of nucleocapsid proteins may serve two functions. Nucleocapsid proteins may have non-structural roles in modulation of nuclear processes in order to maximize virus replication or to inhibit IFN induction and signaling pathways. Alternatively, nucleocapsid proteins may localize coincidentally to the nucleus/nucleolus due to molecular mimicry.

\section{Virus Replication}

The nuclear/nucleolar translocation of the nucleocapsid protein is probably most often associated with optimal virus replication. For example, IBV nucleocapsid redistributes fibrillarin and delays cytokinesis to divert biosynthetic resources from the dividing nucleus to the cytoplasm, where viral replication takes place (Wurm et al., 2001; Chen et al., 2002). The resultant cell cycle arrest/delay would be conducive to higher virus replication through both redirection of biosynthetic machinery for viral replication and delay in cell division. A similar cell cycle arrest has also been observed in other coronaviruses and may be a common consequence of the nucleolar localization of the nucleocapsid protein (Wurm et al., 2001). In nature, these viruses infect terminally differentiated epithelial cells which do not actively divide; it is unclear how the cell cycle modulation observed in cell culture would be useful in a natural infection. The nuclear/nucleolar localization of JEV C protein is also required for optimal virus replication in mammalian cells (Mori et al., 2005). The importance of the nuclear functions of the core protein in flavivirus infection is demonstrated by the inability of some flaviviruses to replicate in enucleated cells (Kos et al., 1975; Lad et al., 1993). Both the Dengue and HCV C protein interact with heterogeneous nuclear RNP K, possibly to regulate host cell transcription, thereby freeing cellular machinery for viral RNA synthesis (Hsieh et al., 1998; Chang et al., 2001).

\section{Interferon Antagonism}

Nucleocapsid proteins of some RNA viruses function in the nucleus to inhibit the potent antiviral IFN response. The innate antiviral response, mediated principally by the action of Type-I IFNs, is one of the earliest responses of the host to viral infection (Conzelmann, 2005). Virus specific intracellular pathogen associated molecular patterns (e.g., viral RNA or dsRNA) are recognized by cellular helicases retinoic-acidinducible protein I and melanoma-differentiation-associated gene 5 (See and Wark, 2008) initiating a cascade of events resulting in the activation of transcription factors including IFN response factor 3 (IRF3), nuclear factor (NF) $\kappa B$ and activating protein 1 that are subsequently transported into the nucleus to activate transcription of IFN- $\beta$. IFN- $\beta$ mRNA is exported out of the nucleus, where it is translated into protein which is secreted from the cell to induce a secondary cellular response in an autocrine and paracrine manner, by binding to the IFN$\alpha / \beta$ receptor. This in turn leads to activation of a second cascade of events involving several effectors and transcription factors such as the STAT proteins. These proteins translocate into the nucleus, interact with IFN-sensitive response elements and activate transcription of a broad range of IFN stimulated genes (ISG). The ISG products mount a concerted immune response which prevents virus replication (See and Wark, 2008).

As mentioned already, MV nucleocapsid protein inhibits the nuclear translocation of STATs, causing disruption of the IFN- $\alpha / \beta$ and IFN- $\gamma$ signaling pathways (Takayama et al., 2012). Coronavirus nucleocapsid protein has been shown to have potent IFN antagonistic activity; however, the mechanism varies in different virus species. The MHV nucleocapsid protein interferes with the IFN induced $2^{\prime}-5^{\prime}$ oligoadenylate synthetase RNase L pathway and can functionally replace the IFN antagonist activity of E3L protein in a recombinant vaccinia virus (Ye et al., 2007), thus contributing to the IFN resistance exhibited by MHV (Rose and Weiss, 2009). The SARS-CoV nucleocapsid protein is also a potent IFN antagonist, however, it is mostly cytoplasmic (discussed above) and inhibits IFN production (Kopecky-Bromberg et al., 2007) 
rather than IFN signaling. Similar to SARS-CoV, the porcine epidemic diarrhea virus nucleocapsid protein inhibits IFN production via direct interaction with TRAF-associated NF-kB activator binding kinase (TBK1), thus interfering with the TBK1 mediated phosphorylation of IRF3, required for IRF3 dimerization and subsequent nuclear localization, which is critical to IFN activation (Zhao, 2013; Ding et al., 2014b). Nucleocapsid protein's IFN antagonist activity may not always be dependent on its nuclear localization.

\section{Molecular Mimicry}

The nucleocapsid protein generally has Lysine $(\mathrm{K})$ or Arginine (R) rich RNA binding domains (to associate with viral genome), which resemble nuclear transport signals and these motifs may serve the dual purpose of mediating nuclear localization of nucleocapsid protein coincidental to their main function. This is the basis for molecular mimicry, wherein the K/Rrich RNA binding domains resembling NLS/NoLS motifs, allow the nucleocapsid protein to be transported into the nucleus by cellular nucleocytoplasmic trafficking machinery (Rowland and Yoo, 2003). Other possibilities include association with ribosomal proteins, and passive diffusion. Association with ribosomal proteins in the cytoplasm might result in nucleocapsid protein being coincidentally translocated into the nucleolus, where the ribosomal proteins associate with rRNA to form ribosomal subunits (Tijms et al., 2002). Although termed "coincidental," nuclear localization of nucleocapsid protein might serve a specific viral purpose by maintaining the nucleocapsid protein:ribonucleotides stoichiometry in the cytoplasm. This is essential in order to produce functional genomes, such that transient nuclear/nucleolar localization of excess nucleocapsid protein can be a means to keep the appropriate concentration of nucleocapsid protein molecules in the cytoplasm (Tijms et al., 2002).

\section{Potential for Future Therapeutic Strategies}

As discussed above, N/NC/C proteins of cytoplasmic RNA viruses have an important nuclear role in addition to their essential cytoplasmic role in virus assembly. This presents an opportunity for a broad ranging antiviral that may inhibit nuclear transport of these proteins, thus indirectly reducing virus assembly, leading to reduced infectious virus production and hence reduced disease. New generations of specific inhibitors of nuclear export (SINE) are well characterized compounds that have low toxicity and good bioavailability and are currently in clinical trials for various cancers and could potentially be re-purposed for virus infections (Azmi et al., 2013a,b; Gao

\section{References}

Ahmed, M., and Lyles, D. S. (1998). Effect of vesicular stomatitis virus matrix protein on transcription directed by host RNA polymerases I, II, and III. J. Virol. 72, 8413-8419. et al., 2014). However, targeting host-pathogen interface is preferable to targeting of cellular processes. Elucidation of the NLS/NoLS motifs of the nucleocapsid proteins and their mechanism of localization should identify interactions that may be modeled for development of small molecule inhibitors (Lundberg et al., 2013); indeed, given the vast array of small molecule inhibitors currently available, it may be possible to identify inhibitors via high throughput screening assays (Wagstaff et al., 2011). Ivermectin and Mifepristone are two such compounds shown to reduce infectious virus titers of several viruses that exploit host nuclear transport machinery (Tay et al., 2013). Determination of the NLS/NoLS also presents an opportunity for development of attenuated vaccine candidates. Specific mutations that disable the nuclear/nucleolar localization function, without hampering the cytoplasmic function of nucleocapsid proteins, should result in viruses that replicate, albeit to lesser degree due to reduced ability to inhibit antiviral responses; mutating the NLS of RSV $M$ protein leads to reduced replication fitness, supporting the viability of such an approach (Ghildyal et al., 2009). That targeting the nucleocapsid protein for attenuation is a viable strategy has been demonstrated for VSV (Sato et al., 2011).

\section{Summary}

Structural proteins of several enveloped RNA viruses localize into the nucleus and/or nucleolus at certain times of the viral life cycle, primarily early in infection. Their nucleocytoplasmic transport is facilitated by nuclear localization/export signals in association with cellular transport proteins. The nucleocapsid protein is a major structural protein of enveloped RNA viruses which serves a structural function in virus assembly. Its function includes protecting the genome against ribonucleases, regulating the fidelity of the replication template, forming the replication complex, and packaging of the genomic RNA for assembly, budding, and particle release. The nucleocapsid proteins of several viruses belonging to Coronaviridae, Arteriviridae, Flaviviridae, and Paramyxoviridae possess NLS/NoLS and transiently localize into the nucleus/nucleolus during the viral life cycle. In some cases the nuclear localization appears to support viral proliferation by interfering with the host's immune response or other mechanisms including maintaining the balance of nucleocapsid protein:RNA molecules for optimum production of the functional viral genome. As such, the nuclear localization of nucleocapsid protein of enveloped RNA viruses has potential as a target for the development of live-attenuated vaccines or antiviral drugs, as inhibition of its nuclear localization could negatively impact on the production of infectious viral particles and cell-to-cell spread. suppress beta interferon gene expression is genetically correlated with the inhibition of host RNA and protein synthesis. J. Virol. 77, 4646-4657. doi: 10.1128/JVI.77.8.4646-4657.2003 
Andersen, J. S., Lyon, C. E., Fox, A. H., Leung, A. K., Lam, Y. W., Steen, H., et al. (2002). Directed proteomic analysis of the human nucleolus. Curr. Biol. 12, 1-11. doi: 10.1016/S0960-9822(01)00650-9

Azmi, A. S., Aboukameel, A., Bao, B., Sarkar, F. H., Philip, P. A., Kauffman, M., et al. (2013a). Selective inhibitors of nuclear export block pancreatic cancer cell proliferation and reduce tumor growth in mice. Gastroenterology 144, 447-456. doi: 10.1053/j.gastro.2012.10.036

Azmi, A. S., Al-Katib, A., Aboukameel, A., Mccauley, D., Kauffman, M., Shacham, S., et al. (2013b). Selective inhibitors of nuclear export for the treatment of non-Hodgkin's lymphomas. Haematologica 98, 1098-1106. doi: 10.3324/haematol.2012.074781

Bartova, E., Horakova, A. H., Uhlirova, R., Raska, I., Galiova, G., Orlova, D., et al. (2010). Structure and epigenetics of nucleoli in comparison with non-nucleolar compartments. J. Histochem. Cytochem. 58, 391-403. doi: 10.1369/jhc.2009.955435

Becker, S., Rinne, C., Hofsass, U., Klenk, H. D., and Muhlberger, E. (1998). Interactions of Marburg virus nucleocapsid proteins. Virology 249, 406-417. doi: 10.1006/viro.1998.9328

Buchholz, C. J., Spehner, D., Drillien, R., Neubert, W. J., and Homann, H. E. (1993). The conserved N-terminal region of Sendai virus nucleocapsid protein NP is required for nucleocapsid assembly. J. Virol. 67, 5803-5812.

Cardarelli, F., Serresi, M., Bizzarri, R., and Beltram, F. (2008). Tuning the transport properties of HIV-1 Tat arginine-rich motif in living cells. Traffic 9, 528-539. doi: 10.1111/j.1600-0854.2007.00696.x

Cerutti, A., Maillard, P., Minisini, R., Vidalain, P. O., Roohvand, F., Pecheur, E. I., et al. (2011). Identification of a functional, CRM-1-dependent nuclear export signal in hepatitis $\mathrm{C}$ virus core protein. PLOS ONE 6:e25854. doi 10.1371/journal.pone.0025854

Chang, C. J., Luh, H. W., Wang, S. H., Lin, H. J., Lee, S. C., and Hu, S. T. (2001). The heterogeneous nuclear ribonucleoprotein K (hnRNP K) interacts with dengue virus core protein. DNA Cell Biol. 20, 569-577. doi: 10.1089/104454901317094981

Chen, H., Wurm, T., Britton, P., Brooks, G., and Hiscox, J. A. (2002). Interaction of the coronavirus nucleoprotein with nucleolar antigens and the host cell. J. Virol. 76, 5233-5250. doi: 10.1128/JVI.76.10.5233-5250.2002

Cohen, S., Au, S., and Pante, N. (2011). How viruses access the nucleus. Biochim. Biophys. Acta 1813, 1634-1645. doi: 10.1016/j.bbamcr.2010.12.009

Conzelmann, K. K. (2005). Transcriptional activation of alpha/beta interferon genes: interference by nonsegmented negative-strand RNA viruses. J. Virol. 79, 5241-5248. doi: 10.1128/JVI.79.9.5241-5248.2005

Curran, J., Marq, J. B., and Kolakofsky, D. (1995). An N-terminal domain of the Sendai paramyxovirus $\mathrm{P}$ protein acts as a chaperone for the NP protein during the nascent chain assembly step of genome replication. J. Virol. 69, 849-855.

de Melo, I. S., Jimenez-Nunez, M. D., Iglesias, C., Campos-Caro, A., MorenoSanchez, D., Ruiz, F. A., et al. (2013). NOA36 protein contains a highly conserved nucleolar localization signal capable of directing functional proteins to the nucleolus, in mammalian cells. PLOS ONE 8:e59065. doi 10.1371/journal.pone.0059065

DiCarlo, A., Moller, P., Lander, A., Kolesnikova, L., and Becker, S. (2007). Nucleocapsid formation and RNA synthesis of Marburg virus is dependent on two coiled coil motifs in the nucleoprotein. Virol. J. 4:105. doi: 10.1186/1743422X-4-105

Ding, L., Huang, Y., Du, Q., Dong, F., Zhao, X., Zhang, W., et al. (2014a). TGEV nucleocapsid protein induces cell cycle arrest and apoptosis through activation of p53 signaling. Biochem. Biophys. Res. Commun. 445, 497-503. doi: 10.1016/j.bbrc.2014.02.039

Ding, Z., Fang, L., Jing, H., Zeng, S., Wang, D., Liu, L., et al. (2014b). Porcine epidemic diarrhea virus nucleocapsid protein antagonizes beta interferon production by sequestering the interaction between IRF3 and TBK1. J. Virol. 88, 8936-8945. doi: 10.1128/JVI.00700-14

Emmott, E., and Hiscox, J. A. (2009). Nucleolar targeting: the hub of the matter. EMBO Rep. 10, 231-238. doi: 10.1038/embor.2009.14

Errington, W., and Emmerson, P. T. (1997). Assembly of recombinant Newcastle disease virus nucleocapsid protein into nucleocapsid-like structures is inhibited by the phosphoprotein. J. Gen. Virol. 78(Pt 9), 2335-2339.

Falcon, V., Acosta-Rivero, N., Chinea, G., De La Rosa, M. C., Menendez, I., Duenas-Carrera, S., et al. (2003). Nuclear localization of nucleocapsidlike particles and $\mathrm{HCV}$ core protein in hepatocytes of a chronically
HCV-infected patient. Biochem. Biophys. Res. Commun. 310, 54-58. doi: 10.1016/j.bbrc.2003.08.118

Fulcher, A. J., and Jans, D. A. (2011). Regulation of nucleocytoplasmic trafficking of viral proteins: an integral role in pathogenesis? Biochim. Biophys. Acta 1813, 2176-2190. doi: 10.1016/j.bbamcr.2011.03.019

Gao, J., Azmi, A. S., Aboukameel, A., Kauffman, M., Shacham, S., Abou-Samra, A. B., et al. (2014). Nuclear retention of Fbw7 by specific inhibitors of nuclear export leads to Notch1 degradation in pancreatic cancer. Oncotarget 5, 34443454 .

Ghildyal, R., Ho, A., Dias, M., Soegiyono, L., Bardin, P. G., Tran, K. C., et al. (2009). The respiratory syncytial virus matrix protein possesses a Crm1mediated nuclear export mechanism. J. Virol. 83, 5353-5362. doi: 10.1128/JVI. 02374

Ghildyal, R., Ho, A., Wagstaff, K. M., Dias, M. M., Barton, C. L., Jans, P., et al. (2005). Nuclear import of the respiratory syncytial virus matrix protein is mediated by importin betal independent of importin alpha. Biochemistry 44, 12887-12895. doi: 10.1021/bi050701e

Golomb, L., Volarevic, S., and Oren, M. (2014). p53 and ribosome biogenesis stress: the essentials. FEBS Lett. 588, 2571-2579. doi: 10.1016/j.febslet.2014.04.014

Gorlich, D., and Kutay, U. (1999). Transport between the cell nucleus and the cytoplasm. Annu. Rev. Cell Dev. Biol. 15, 607-660. doi: 10.1146/annurev.cellbio.15.1.607

Green, T. J., Rowse, M., Tsao, J., Kang, J., Ge, P., Zhou, Z. H., et al. (2011). Access to RNA encapsidated in the nucleocapsid of vesicular stomatitis virus. J. Virol. 85, 2714-2722. doi: 10.1128/JVI.01927-10

Heinrich, B. S., Morin, B., Rahmeh, A. A., and Whelan, S. P. (2012). Structural properties of the $\mathrm{C}$ terminus of vesicular stomatitis virus $\mathrm{N}$ protein dictate N-RNA complex assembly, encapsidation, and RNA synthesis. J. Virol. 86, 8720-8729. doi: 10.1128/JVI.00990-12

Hiscox, J. A. (2002). The nucleolus-a gateway to viral infection? Arch. Virol. 147, 1077-1089. doi: 10.1007/s00705-001-0792-0

Hiscox, J. A., Wurm, T., Wilson, L., Britton, P., Cavanagh, D., and Brooks, G. (2001). The coronavirus infectious bronchitis virus nucleoprotein localizes to the nucleolus. J. Virol. 75, 506-512. doi: 10.1128/JVI.75.1.506-512.2001

Hsieh, T. Y., Matsumoto, M., Chou, H. C., Schneider, R., Hwang, S. B. Lee, A. S., et al. (1998). Hepatitis C virus core protein interacts with heterogeneous nuclear ribonucleoprotein K. J. Biol. Chem. 273, 17651-17659. doi: $10.1074 /$ jbc.273.28.17651

Hunter, E. (2007). "Virus assembly," in Fields Virology, eds P. M. Howley and D. M. Knipe (Philadelphia: Lippincott Williams), 141-168.

Jones, D. M., Atoom, A. M., Zhang, X., Kottilil, S., and Russell, R. S. (2011) A genetic interaction between the core and NS3 proteins of hepatitis C virus is essential for production of infectious virus. J. Virol. 85, 12351-12361. doi: 10.1128/JVI.05313-11

Khattar, S. K., Yunus, A. S., Collins, P. L., and Samal, S. K. (2000). Mutational analysis of the bovine respiratory syncytial virus nucleocapsid protein using a minigenome system: mutations that affect encapsidation, RNA synthesis, and interaction with the phosphoprotein. Virology 270, 215-228. doi: 10.1006/viro.2000.0264

Kopecky-Bromberg, S. A., Martinez-Sobrido, L., Frieman, M., Baric, R. A., and Palese, P. (2007). Severe acute respiratory syndrome coronavirus open reading frame (ORF) 3b, ORF 6, and nucleocapsid proteins function as interferon antagonists. J. Virol. 81, 548-557. doi: 10.1128/JVI. 01782-06

Kos, K. A., Osborne, B. A., and Goldsby, R. A. (1975). Inhibition of group $\mathrm{B}$ arbovirus antigen production and replication in cells enucleated with cytochalasin B. J. Virol. 15, 913-917.

Lad, V. J., Gupta, A. K., Ghosh, S. N., and Banerjee, K. (1993). Immunofluorescence studies on the replication of some arboviruses in nucleated and enucleated cells. Acta Virol. 37, 79-83.

Lam, Y. W., Trinkle-Mulcahy, L., and Lamond, A. I. (2005). The nucleolus. J. Cell Sci. 118, 1335-1337. doi: 10.1242/jcs.01736

Lee, C., Hodgins, D., Calvert, J. G., Welch, S. K., Jolie, R., and Yoo, D. (2006). Mutations within the nuclear localization signal of the porcine reproductive and respiratory syndrome virus nucleocapsid protein attenuate virus replication. Virology 346, 238-250. doi: 10.1016/j.virol.2005.11.005

Longhi, S. (2009). Nucleocapsid structure and function. Curr. Top. Microbiol. Immunol. 329, 103-128. doi: 10.1007/978-3-540-70523-9_6 
Lundberg, L., Pinkham, C., Baer, A., Amaya, M., Narayanan, A., Wagstaff, K. M., et al. (2013). Nuclear import and export inhibitors alter capsid protein distribution in mammalian cells and reduce Venezuelan Equine Encephalitis Virus replication. Antiviral Res. 100, 662-672. doi: 10.1016/j.antiviral.2013.10.004

Lynch, S., and Kolakofsky, D. (1978). Ends of the RNA within Sendai virus defective interfering nucleocapsids are not free. J. Virol. 28, 584-589.

Macara, I. G. (2001). Transport into and out of the nucleus. Microbiol. Mol. Biol. Rev. 65, 570-594. doi: 10.1128/MMBR.65.4.570-594.2001

McBride, R., Van Zyl, M., and Fielding, B. C. (2014). The coronavirus nucleocapsid is a multifunctional protein. Viruses 6, 2991-3018. doi: 10.3390/v6082991

Mir, M. A., and Panganiban, A. T. (2006). The bunyavirus nucleocapsid protein is an RNA chaperone: possible roles in viral RNA panhandle formation and genome replication. RNA 12, 272-282. doi: 10.1261/rna. 2101906

Mohammadi, H., Sharif, S., Rowland, R. R., and Yoo, D. (2009). The lactate dehydrogenase-elevating virus capsid protein is a nuclear-cytoplasmic protein. Arch. Virol. 154, 1071-1080. doi: 10.1007/s00705-009-0410-0

Mori, Y., Okabayashi, T., Yamashita, T., Zhao, Z., Wakita, T., Yasui, K., et al. (2005). Nuclear localization of Japanese encephalitis virus core protein enhances viral replication. J. Virol. 79, 3448-3458. doi: 10.1128/JVI.79.6.3448-3458.2005

Morin, B., and Whelan, S. P. (2014). Sensitivity of the polymerase of vesicular stomatitis virus to 2' substitutions in the template and nucleotide triphosphate during initiation and elongation. J. Biol. Chem. 289, 9961-9969. doi: 10.1074/jbc.M113.542761

Myers, T. M., and Moyer, S. A. (1997). An amino-terminal domain of the Sendai virus nucleocapsid protein is required for template function in viral RNA synthesis. J. Virol. 71, 918-924.

Myers, T. M., Smallwood, S., and Moyer, S. A. (1999). Identification of nucleocapsid protein residues required for Sendai virus nucleocapsid formation and genome replication. J. Gen. Virol. 80(Pt 6), 1383-1391.

Pei, Y., Hodgins, D. C., Lee, C., Calvert, J. G., Welch, S. K., Jolie, R., et al. (2008). Functional mapping of the porcine reproductive and respiratory syndrome virus capsid protein nuclear localization signal and its pathogenic association. Virus Res. 135, 107-114. doi: 10.1016/j.virusres.2008. 02.012

Rajkowitsch, L., Chen, D., Stampfl, S., Semrad, K., Waldsich, C., Mayer, O., et al. (2007). RNA chaperones, RNA annealers and RNA helicases. RNA Biol. 4, 118-130. doi: 10.4161/rna.4.3.5445

Raymond, D. D., Piper, M. E., Gerrard, S. R., and Smith, J. L. (2010). Structure of the Rift Valley fever virus nucleocapsid protein reveals another architecture for RNA encapsidation. Proc. Natl. Acad. Sci. U.S.A. 107, 11769-11774. doi: 10.1073/pnas.1001760107

Reed, M. L., Dove, B. K., Jackson, R. M., Collins, R., Brooks, G., and Hiscox, J. A. (2006). Delineation and modelling of a nucleolar retention signal in the coronavirus nucleocapsid protein. Traffic 7, 833-848. doi: 10.1111/j.16000854.2006.00424.x

Robbins, S. J., and Eagle, J. P. (1985). Enhanced intranuclear expression of measles virus following exposure of persistently infected cells to cyclic AMP. J. Gen. Virol. 66(Pt 9), 2065-2070. doi: 10.1099/0022-1317-66-9-2065

Rose, K. M., and Weiss, S. R. (2009). Murine coronavirus cell type dependent interaction with the type I interferon response. Viruses 1, 689-712. doi: 10.3390/v1030689

Rowland, R. R., Kervin, R., Kuckleburg, C., Sperlich, A., and Benfield, D. A. (1999). The localization of porcine reproductive and respiratory syndrome virus nucleocapsid protein to the nucleolus of infected cells and identification of a potential nucleolar localization signal sequence. Virus Res. 64, 1-12. doi: 10.1016/S0168-1702(99)00048-9

Rowland, R. R., and Yoo, D. (2003). Nucleolar-cytoplasmic shuttling of PRRSV nucleocapsid protein: a simple case of molecular mimicry or the complex regulation by nuclear import, nucleolar localization and nuclear export signal sequences. Virus Res. 95, 23-33. doi: 10.1016/S0168-1702(03)00161-8

Sangiambut, S., Keelapang, P., Aaskov, J., Puttikhunt, C., Kasinrerk, W., Malasit, P., et al. (2008). Multiple regions in dengue virus capsid protein contribute to nuclear localization during virus infection. J. Gen. Virol. 89, 1254-1264. doi: 10.1099/vir.0.83264-0

Sato, H., Masuda, M., Miura, R., Yoneda, M., and Kai, C. (2006). Morbillivirus nucleoprotein possesses a novel nuclear localization signal and a
CRM1-independent nuclear export signal. Virology 352, 121-130. doi: 10.1016/j.virol.2006.04.013

Sato, H., Yoneda, M., Honda, T., and Kai, C. (2011). Recombinant vaccines against the mononegaviruses-what we have learned from animal disease controls. Virus Res. 162, 63-71. doi: 10.1016/j.virusres.2011. 09.038

Schnell, M. J., Mcgettigan, J. P., Wirblich, C., and Papaneri, A. (2010). The cell biology of rabies virus: using stealth to reach the brain. Nat. Rev. Microbiol. 8, 51-61. doi: 10.1038/nrmicro2260

See, H., and Wark, P. (2008). Innate immune response to viral infection of the lungs. Paediatr. Respir. Rev. 9, 243-250. doi: 10.1016/j.prrv.2008. 04.001

Sharma, K., Didier, P., Darlix, J. L., De Rocquigny, H., Bensikaddour, H., Lavergne, J. P., et al. (2010). Kinetic analysis of the nucleic acid chaperone activity of the hepatitis C virus core protein. Nucleic Acids Res. 38, 3632-3642. doi: 10.1093/nar/gkq094

Shaw, P., and Brown, J. (2012). Nucleoli: composition, function, and dynamics. Plant Physiol. 158, 44-51. doi: 10.1104/pp.111.188052

Sun, Y., Guo, Y., and Lou, Z. (2012). A versatile building block: the structures and functions of negative-sense single-stranded RNA virus nucleocapsid proteins. Protein Cell 3, 893-902. doi: 10.1007/s13238-012-2087-5

Surjit, M., Kumar, R., Mishra, R. N., Reddy, M. K., Chow, V. T., and Lal, S. K. (2005). The severe acute respiratory syndrome coronavirus nucleocapsid protein is phosphorylated and localizes in the cytoplasm by 14-3-3-mediated translocation. J. Virol. 79, 11476-11486. doi: 10.1128/JVI.79.17.1147611486.2005

Suzuki, R., Sakamoto, S., Tsutsumi, T., Rikimaru, A., Tanaka, K., Shimoike, T., et al. (2005). Molecular determinants for subcellular localization of hepatitis C virus core protein. J. Virol. 79, 1271-1281. doi: 10.1128/JVI.79.2.12711281.2005

Takayama, I., Sato, H., Watanabe, A., Omi-Furutani, M., Sugai, A., Kanki, K., et al. (2012). The nucleocapsid protein of measles virus blocks host interferon response. Virology 424, 45-55. doi: 10.1016/j.virol.2011. 12.011

Tay, M. Y., Fraser, J. E., Chan, W. K., Moreland, N. J., Rathore, A. P., Wang, C., et al. (2013). Nuclear localization of dengue virus (DENV) 1-4 non-structural protein 5; protection against all $4 \mathrm{DENV}$ serotypes by the inhibitor Ivermectin. Antiviral Res. 99, 301-306. doi: 10.1016/j.antiviral.2013.06.002

Tijms, M. A., Van Der Meer, Y., and Snijder, E. J. (2002). Nuclear localization of non-structural protein 1 and nucleocapsid protein of equine arteritis virus. J. Gen. Virol. 83, 795-800.

Tsuda, Y., Mori, Y., Abe, T., Yamashita, T., Okamoto, T., Ichimura, T., et al. (2006). Nucleolar protein B23 interacts with Japanese encephalitis virus core protein and participates in viral replication. Microbiol. Immunol. 50, 225-234. doi: 10.1111/j.1348-0421.2006. tb03789.x

Urbanowski, M. D., Ilkow, C. S., and Hobman, T. C. (2008). Modulation of signaling pathways by RNA virus capsid proteins. Cell. Signal. 20, 1227-1236. doi: 10.1016/j.cellsig.2007.12.018

Verheggen, C., and Bertrand, E. (2012). CRM1 plays a nuclear role in transporting snoRNPs to nucleoli in higher eukaryotes. Nucleus 3, 132-137. doi: 10.4161/nucl.19266

Wagstaff, K. M., Rawlinson, S. M., Hearps, A. C., and Jans, D. A. (2011). An AlphaScreen(R)-based assay for high-throughput screening for specific inhibitors of nuclear import. J. Biomol. Screen. 16, 192-200. doi: $10.1177 / 1087057110390360$

Wang, Y. E., Park, A., Lake, M., Pentecost, M., Torres, B., Yun, T. E., et al. (2010). Ubiquitin-regulated nuclear-cytoplasmic trafficking of the Nipah virus matrix protein is important for viral budding. PLoS Pathog. 6:e1001186. doi: 10.1371/journal.ppat.1001186

Whelan, S. P. (2013). "Viral Replication Strategies," in Fields Virology, 6th Edn, eds D. M. Knipe and P. M. Howley (Philadelphia: Lippincott Williams \& Wilkins), 105-126.

Wurm, T., Chen, H., Hodgson, T., Britton, P., Brooks, G., and Hiscox, J. A. (2001). Localization to the nucleolus is a common feature of coronavirus nucleoproteins, and the protein may disrupt host cell division. J. Virol. 75, 9345-9356. doi: 10.1128/JVI.75.19.93459356.2001 
Ye, Y., Hauns, K., Langland, J. O., Jacobs, B. L., and Hogue, B. G. (2007). Mouse hepatitis coronavirus A59 nucleocapsid protein is a type I interferon antagonist. J. Virol. 81, 2554-2563. doi: 10.1128/JVI.01634-06

Yoo, D., Wootton, S. K., Li, G., Song, C., and Rowland, R. R. (2003). Colocalization and interaction of the porcine arterivirus nucleocapsid protein with the small nucleolar RNA-associated protein fibrillarin. J. Virol. 77, 12173-12183. doi: 10.1128/JVI.77.22.12173-12183.2003

You, J., Dove, B. K., Enjuanes, L., Dediego, M. L., Alvarez, E., Howell, G., et al. (2005). Subcellular localization of the severe acute respiratory syndrome coronavirus nucleocapsid protein. J. Gen. Virol. 86, 3303-3310. doi: 10.1099/vir.0.81076-0

You, J. H., Reed, M. L., and Hiscox, J. A. (2007). Trafficking motifs in the SARS-coronavirus nucleocapsid protein. Biochem. Biophys. Res. Commun. 358, 1015-1020. doi: 10.1016/j.bbrc.2007.05.036

Zhang, X., Green, T. J., Tsao, J., Qiu, S., and Luo, M. (2008). Role of intermolecular interactions of vesicular stomatitis virus nucleoprotein in RNA encapsidation. J. Virol. 82, 674-682. doi: 10.1128/JVI.00935-07
Zhao, W. (2013). Negative regulation of TBK1-mediated antiviral immunity. FEBS Lett. 587, 542-548. doi: 10.1016/j.febslet.2013.01.052

Zuniga, S., Sola, I., Moreno, J. L., Sabella, P., Plana-Duran, J., and Enjuanes, L. (2007). Coronavirus nucleocapsid protein is an RNA chaperone. Virology 357, 215-227. doi: 10.1016/j.virol.2006.07.046

Conflict of Interest Statement: The authors declare that the research was conducted in the absence of any commercial or financial relationships that could be construed as a potential conflict of interest.

Copyright (c) 2015 Wulan, Heydet, Walker, Gahan and Ghildyal. This is an openaccess article distributed under the terms of the Creative Commons Attribution License (CC BY). The use, distribution or reproduction in other forums is permitted, provided the original author(s) or licensor are credited and that the original publication in this journal is cited, in accordance with accepted academic practice. No use, distribution or reproduction is permitted which does not comply with these terms. 